Gynäkol Rundsch 1989;29(Suppl 1):I-IV

\title{
Contents, Vol. 29, Supplement 1, 1989
}

Inhalt

Vorwort

1

Zur Emeritierung von Prof. W.E. Schreiner

Keller, P.J 2

Dem Leben verpflichtet

Böckle, F 4

Die Bedeutung von sekundären Operationen beim Ovarialkarzinom

Engeler, V

17

Inguinale Lymphome mit Beinschwellung als Leitsymptome des

Tubenkarzinoms

Baer, S.; Schreiner, W.E 23

Mammakarzinom: Ein diagnostisches oder therapeutisches Problem?

Schmid,J

29

Brusterhaltende Therapie des primären Mammakarzinoms.

Resultate der Universitäts-Frauenklinik Zurich

Steiner, R.A.; Keller, K.; Engeler, V.; Schreiner, W.E 35

Reflexionen über die abdominale erweiterte Hysterektomie

Stoll,W

44

Gynäkologische Operationen bei der älteren Patientin

Bronz, L 55

Veränderungen urodynamischer Parameter nach Zystourethropexie

Wight, E.; Müller, R.; Schreiner, W.E

Immunologie der Schwangerschaft. Neuere Erkenntnisse

Maroni, E

63

Inhalt IV

1st der Fortschritt in der Geburtshilfe messbar?

Bretscher, J 68

Universitätsspital und Schwerpunktspital:

Ein interaktives Ausbildungssystem

Neuenschwander, F 71

Die gynäkologische Klinik im Zeitalter der EDV

Rageth,J.C 75

35 Jahre Frauenheilkunde - Rückblick und Ausblick

Schreiner, W.E

81

Autorenregister und Donatoren 